Crystal Electric Field Effects and Thermal Expansion of RE- Hexaborides

V.V. Novikov ${ }^{1}$, E.S. Pilipenko ${ }^{1}$, S.L.Bud'ko ${ }^{2}$

(C) 2017. This manuscript version is made available under the Elsevier user license http://www.elsevier.com/open-access/userlicense/1.0/ 


\title{
Crystal Electric Field Effects and Thermal Expansion of Rare-Earth
}

\section{Hexaborides}

\begin{abstract}
Anomalies in the magnetic contribution to the thermal expansion coefficients $\Delta \beta(T)$ of the $\mathrm{CeB}_{6}, \mathrm{PrB}_{6}$, and $\mathrm{NdB}_{6}$ hexaborides in the range of 5-300 K were found by comparison with diamagnetic $\mathrm{LaB}_{6}$. The characteristic of the anomalies was the same in all the studied borides: a distinct peak at low temperatures, followed by a broad maximum at higher temperatures $(50-100 \mathrm{~K})$, then a decrease and transition to the region of negative values as the temperature increases further. The features of $\Delta \beta(T)$ are explained by the effects of the magnetic order (sharp low temperature peaks) and the crystal electric field (CEF). The $\beta_{C E F}(T)$ dependencies were calculated using Raman and neutron scattering data on the splitting of the rare-earth (RE) ions $\mathrm{R}^{3+}$ f-level by the CEF. A satisfactory consistency between the values of $\beta_{C E F}(T)$ and $\Delta \beta(T)$ was obtained for the studied hexaborides. Additionally, we determined the values of the Grüneisen parameter $\gamma_{\mathrm{i}}$ that correspond to the transition between the ground and excited multiplets of $\mathrm{R}^{3+}$ ions f-level splitting.
\end{abstract}

Keywords: hexaborides, thermal expansion, crystal electric field, low temperatures

\section{Introduction}


Hexaborides of rare-earth (RE) elements $\left(\mathrm{RB}_{6}\right.$, where $\mathrm{R}$ stands for the $\mathrm{RE}$ metal) are a group of compounds whose properties have recently received considerable attention. Most work dedicated to studying the properties of RE hexaborides has dealt with different aspects of the transitions of their magnetic subsystems into an ordered state when the temperature decreases [1-10] as well as their electronic properties. [11-14] Calorimetric studies, [15-25] including those in which high magnetic fields were used, [2,6] have enabled to reveal the characteristic of the ground state of $\mathrm{R}^{3+}$ ions in hexaborides, the type of magnetic ordering, and the thermodynamic characteristics (enthalpy and entropy) of phase transformations. Studies of the thermal expansion of RE hexaborides [23,25-27] revealed sharp anomalies in the temperature dependence of the lattice parameters at the phase transition temperatures $(5-30 \mathrm{~K})$ and broader anomalies at higher temperatures (50-300 K). In this study, we address the nature of the thermal expansion anomalies in RE hexaborides. We have investigated the effect of magnetic phase transitions and crystal electric field (CEF) on thermal expansion of the hexaborides using the experimental data obtained earlier with the participation of one of the authors (V.V.N.) of the present paper.

\section{Theory}

Let us consider a system of non-interacting RE ions with the ground state split by a CEF. [28,29] 
The statistic sum for the CEF-multiplets system with energies of $E_{i}$ and degeneracy multiplicity of $g_{i}$ is as follows:

$$
\begin{aligned}
& Z=\sum_{i} g_{i} \exp \left(-E_{i} / k_{B} T\right) . \\
& F=-k_{B} T \ln Z
\end{aligned}
$$

defines system free energy $F$.

Here $N$ is the number of rare earth ions per unit volume, $k_{B}$ is the Boltzmann constant. The relationship between free energy $F$ and the volumetric thermal expansion coefficient $\beta$ is

$$
\beta=-\kappa \frac{\partial^{2} F}{\partial V \partial T},
$$

where $\kappa$ stands for the isothermal compressibility.

We introduce the CEF Grüneisen parameter:

$$
\gamma_{i C E F}=\frac{\partial \ln E i}{\partial \ln V} .
$$

Substituting (1) in (2), differentiating $F$ in volume and temperature, and taking into account (4), we obtain:

$$
\beta=\frac{\kappa N}{k_{B} T}\left[<E^{2} \gamma>-<E \gamma><E>\right] .
$$

In Equation (4) $E$ is the value of CEF-splitting, and the angular brackets $<>$ denote the average thermodynamic values:

$$
<X>=\frac{\sum_{i} X_{i} \exp \left(-E_{i} / k_{B} T\right)}{\sum_{i} g_{i} \exp \left(-E_{i} / k_{B} T\right)}
$$


Thus, the influence of CEF leads to a supplementary contribution to the thermal expansion. Depending on the $\mathrm{E}_{i}$ values and the $\gamma_{i \text { CEF }}$ Grüneisen parameter, the $\mathrm{CEF}$ can elevate or reduce $\beta(T)$ in different parts of the temperature range.

\section{Results and discussion}

We analysed the results of $\mathrm{x}$-ray studies on the lattice parameters for $\mathrm{LaB}_{6}$, $\mathrm{CeB}_{6}, \mathrm{PrB}_{6}$, and $\mathrm{NdB}_{6}$ in the temperature range of $5-300 \mathrm{~K}[23,27]$ using the schemes of splitting the ground f-level RE ions in hexaborides obtained by Raman and neutron spectroscopy. [28,29]

Figure 1 shows the experimental temperature dependencies of unit cell volume $\mathrm{V}(\mathrm{T})$ in the $\mathrm{RB}_{6}$ hexaborides $(\mathrm{RE}=\mathrm{La}, \mathrm{Ce}, \mathrm{Pr}$, and $\mathrm{Nd})$. [23,27]

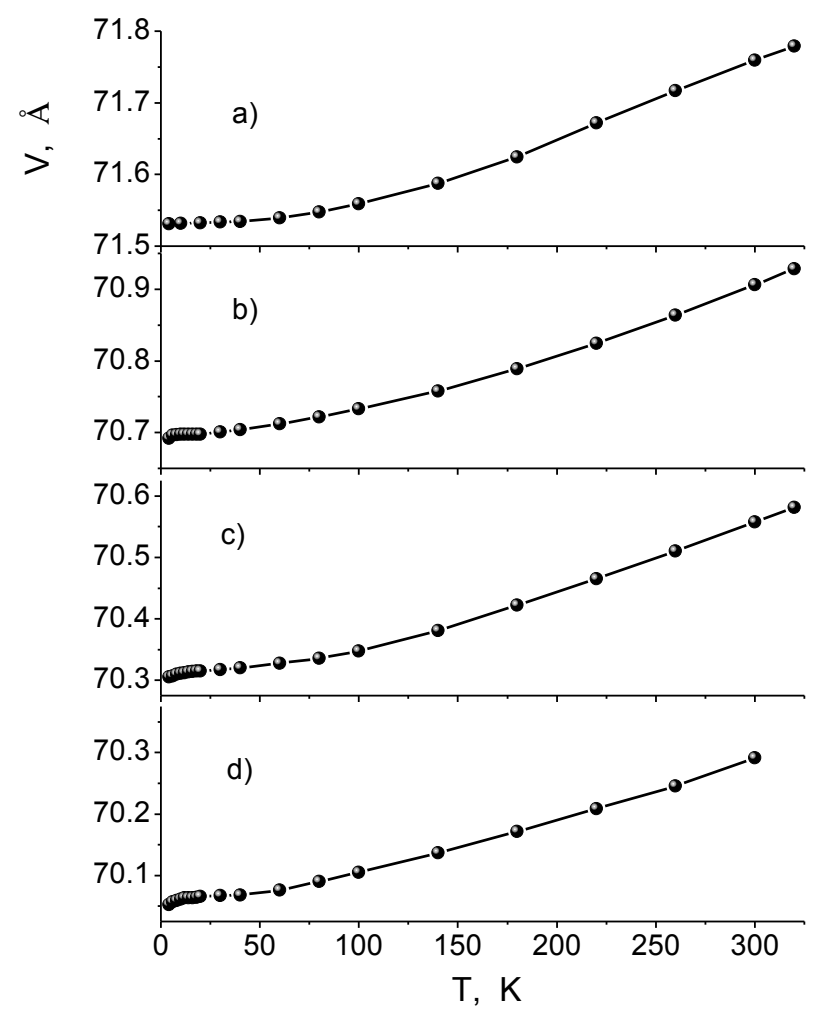

Figure 1. Unit cell volume of RE hexaborides; $a: \mathrm{LaB}_{6} ; \mathrm{b}: \mathrm{CeB}_{6} ; \mathrm{c}: \mathrm{PrB}_{6}$; and d: $\mathrm{NdB}_{6} \cdot[27]$ 
Figure 2 shows the thermal expansion anomalies more clearly. In particular, we show the estimate of the magnetic contribution $\Delta \beta_{R B_{6}}(T)$, which is defined as the difference between the volume thermal expansion coefficients of the RE hexaborides and diamagnetic lanthanum hexaboride, to the volume thermal expansion:

$$
\begin{gathered}
\beta_{R B_{6}}(T)=\left(1 / V_{R B_{6}}\right)\left(d V_{R B_{6}}(T) / d T\right) \text { and } \\
\Delta \beta_{R B_{6}}(T)=\beta_{R B_{6}}(T)-\beta_{L a B_{6}}(T) .
\end{gathered}
$$

Since the value of $\Delta \beta_{R B_{6}}(T)$ is the difference between the two close values $\beta_{R B_{6}}(T)$ and $\beta_{L a B_{6}}(T)$, the experimental error of $\Delta \beta_{R B_{6}}(T)$ is rather large and according to our estimates is about $40 \%$.

(a)

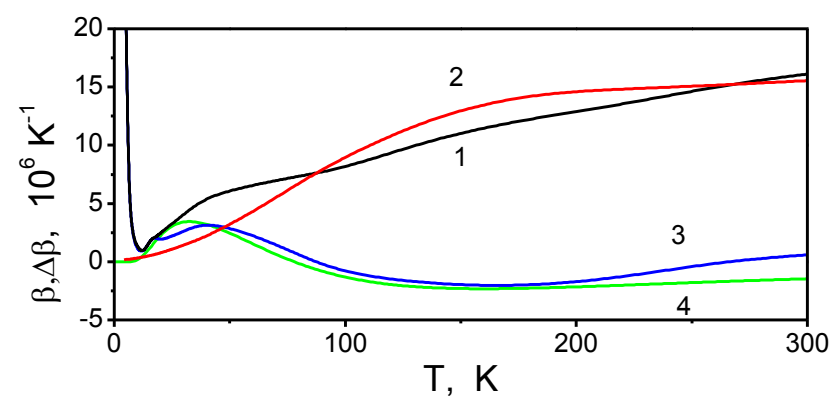

(b)

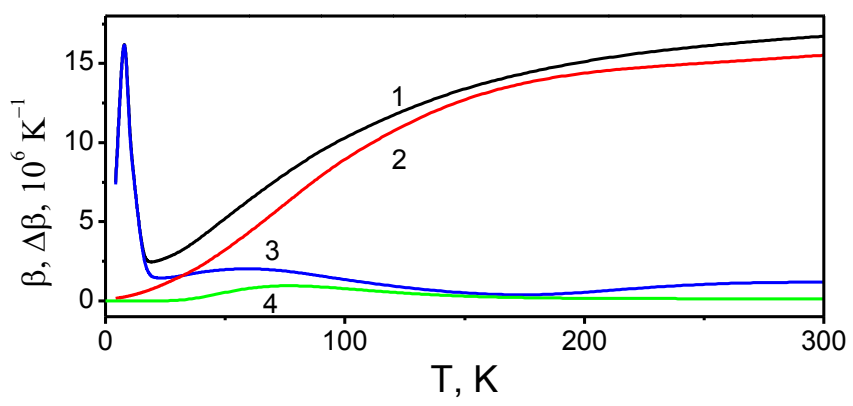

(c) 


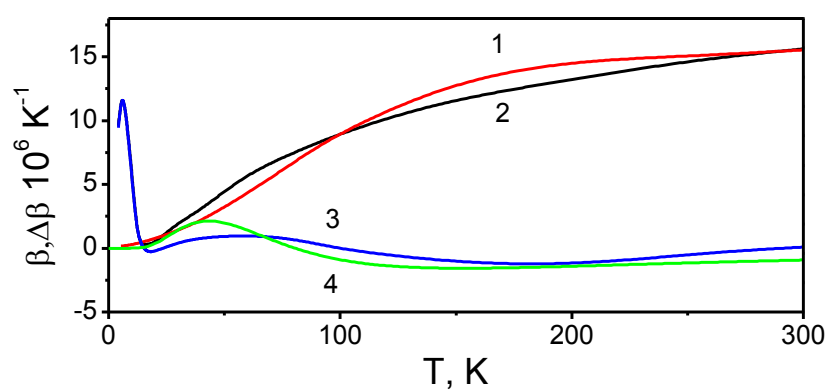

Figure 2. Thermal expansion characteristics of RE hexaborides: (a) $\mathrm{CeB}_{6}$; (b) $\mathrm{PrB}_{6}$; and (c) $\mathrm{NdB}_{6} .1$ : Volume thermal expansion coefficient $\beta_{R B_{6}}(T)(\mathrm{R}=\mathrm{Ce}, \mathrm{Pr}$, and Nd). 2: $\beta_{L A B_{6}}(T) .3: \Delta \beta_{R B_{6}}(T) .4: \Delta \beta_{C E F}(T)$.

Apparently, the sharp low-temperature peaks $\Delta \beta_{R B_{6}}(T)$ are due to magnetic phase transitions in hexaborides $\left(T_{N C E B_{6}}=2.3 \mathrm{~K} ; T_{N \mathrm{Pr}_{6}}=6.9 \mathrm{~K} ; T_{N N d B_{6}}=8.6 \mathrm{~K}\right)$. $[1,6,8,9]$

We assume that the higher temperature broad anomalies of $\Delta \beta_{R B_{6}}(T)$ (the maxima being about $30-50 \mathrm{~K}$, the minima being about $100-200 \mathrm{~K}$ ) could be understood as the effect of CEF on $\mathrm{R}^{3+}$ ion f-levels in the hexaborides. To verify this hypothesis, we used data on CEF-splitting for $\mathrm{CeB}_{6}$, [30] $\mathrm{PrB}_{6}$, and $\mathrm{NdB}_{6}$ [31] (Figure 3).

a)

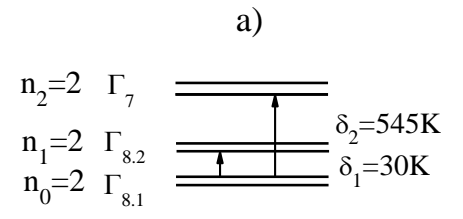

b)

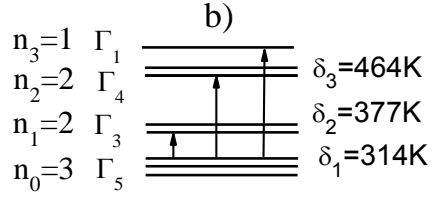

c)

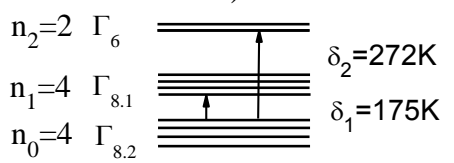

Figure 3. Schemes of CEF-splitting of hexaboride f-levels: a) $\mathrm{CeB}_{6}$ [30]; b) $\mathrm{PrB}_{6}$ [31]; and c) $\mathrm{NdB}_{6}$. [31] 
In accordance with the scheme of CEF-splitting of $\mathrm{R}^{3+}$ ion f-levels in hexaborides, the statistical sums for $\mathrm{CeB}_{6}, \mathrm{PrB}_{6}$, and $\mathrm{NdB}_{6}$ are as follows:

$$
\left\{\begin{array}{l}
Z_{C B_{6}}=2 \exp \left(-\delta_{0} / T\right)+2 \exp (-30 / T)+2 \exp (-545 / T) \\
Z_{\mathrm{Pr}_{B_{6}}}=3 \exp \left(-\delta_{0} / T\right)+2 \exp (-314 / T)+2 \exp (-377 / T)+\exp (-464 / T) \\
Z_{N d B_{6}}=4 \exp \left(\delta_{0} / T\right)+4 \exp (-175 / T)+2 \exp (-278 / T)
\end{array}\right.
$$

The ground multiplet energy is assumed to be $\delta_{0}=0$.

By simultaneously solving Equations (4), (5), and (9), using $\gamma_{\mathrm{i}}$ as a fitting parameter, it is possible to calculate the $\Delta \beta_{C E F}(T)$ of the studied hexaborides (see line 4 in Figure 2). We obtained the next values of $\gamma_{\mathrm{i}}: \mathrm{CeB}_{6}-\gamma_{1}=7, \gamma_{2}=8$; $\operatorname{PrB}_{6}-$ $\gamma_{1}=22, \gamma_{2}=-23, \gamma_{3}=30 ;$ and $\mathrm{NdB}_{6}-\gamma_{1}=6, \gamma_{2}=-15$

As shown in Figure 3, the calculated dependencies $\Delta \beta_{C E F}(T)$ satisfactorily reproduce the temperature behaviour of the $\Delta \beta_{R B_{6}}(T)$ curves. The noticeable differences between the experimental and calculated curves for $\operatorname{PrB}_{6}$ and $\mathrm{NdB}_{6}$ can be explained by the different qualities of the hexaboride samples that were used in [30,31] and in the present work, as well as by errors in calculating the thermal expansion coefficient values. The temperatures of maxima in $\Delta \beta_{R B_{6}}(T)$ and $\Delta \beta_{C E F}(T)$ are correlated with the corresponding $\delta_{1}$ splitting values (Figure 4).

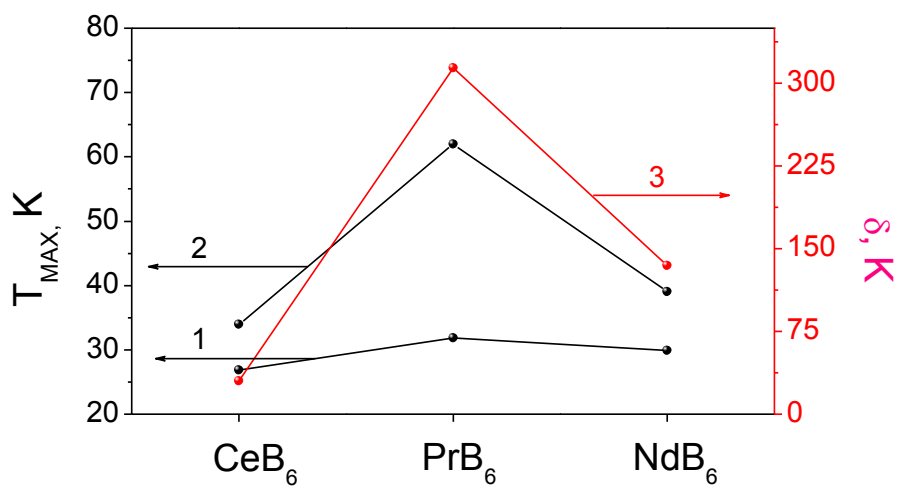


Figure 4. The temperatures of maxima of $\Delta \beta_{R B_{6}}(T)(1), \Delta \beta_{C E F}(T)(2)$, and $\delta_{1}$ splitting values (3) for the borides studied.

Thus, the following conclusion can be drawn: the high-temperature anomalies of $\mathrm{CeB}_{6}, \mathrm{PrB}_{6}$, and $\mathrm{NdB}_{6}$ hexaboride thermal expansion are due to the effect of CEF on $\mathrm{R}^{3+}$ ions system.

Large values of the Grüneisen parameter $\gamma_{i C E F}$ (up to several tens) and the same distribution of signs in $\gamma_{\text {CCEF }}$ for all compounds in the studied group are the characteristic features of $\mathrm{CEF}$ effects on $\mathrm{RB}_{6}$ thermal expansion. We conclude that $\gamma_{1 C E F}>0$, a positive contribution to the thermal expansion, corresponds to the transition to the first excited multiplet, whereas $\gamma_{2 C E F}<0$, a negative contribution to the $\beta_{R B_{6}}(T)$ values, corresponds to the transition to the next multiplet.

\section{Conclusions}

The CEF has a significant effect on the magnetic, electric, and thermal properties of a material in a wide range of low temperatures. The present study is one of the first attempt to determine a reasons which lead to thermal expansion anomalies of RE-hexaborides at elevated temperatures. It was found that the experimentally identified anomalous regularities of the temperature dependences of the thermal expansion of rare-earth hexaborides at moderately low temperatures can be due to CEF effect on the system of $\mathrm{R}^{3+}$ paramagnetic ions. CEF-splitting of the f-levels in RE hexaborides leads to the Schottky heat capacity contribution and 
to thermal expansion anomalies. Deviations of thermal characteristics of RE hexaborides from their regular (lattice) values can be calculated using the established schemes of CEF-splitting. The approach used in this article can be applied to analyze the anomalies of thermal properties of magnets of different classes.

\section{Acknowledgements}

The research was performed under the auspices of the Ministry of Education and Science of the Russian Federation (State assignment, project № 3.1049.2017/PCh). Work at the Ames Laboratory (S.L.B.) was supported by the U.S. Department of Energy, Office of Basic Energy Science, Division of Materials Sciences and Engineering under Contract No. DE-AC0207CH11358.

\section{References}

[1] B.T. Matthias, T.H. Geballe, K. Andress, E. Gorenzwit, G.W. Hull, J.P. Maita, Science 159530 (1968)

[2] J.M. Effantin, J. Rossat-Mignod, P. Burlet, H. Bartholin, S. Kunii, T. Kasuya, J. Magn. Magn. Mater. 47,48 145-148 (1985)

[3] J.C. Nickerson, R.M. White, K.N. Lee, R. Bachman, T.H. Geballe, G.W. Hull, Phys. Rev. B. 3 2030-2042 (1971)

[4] J.C Cooley, M.C Aronson, J.L. Sarrao, Z.Fisk, Physical Review B. 56 14541$14546(1997)$ 
[5] Kunii, K. Iwashita, T. Matsumura, K. Segawa, Physica B. 186-188 646-648 (1993)

[6] T. Fugita, M. Suzuki, T. Komatsubara, S. Kunii, T. Kasuya, T. Ohtsuka, Sol. State Commun. 35 569-572 (1980)

[7] N. Shino, S. Suga, S. Imada, Y. Saitoh, H. Yamada, T. Namba, S. Kimura, S. Kunii, J. Phys. Soc. Jpn. 64 2980-2987 (1995)

[8] C.M. McCarthy, C.W. Tompson, R.J. Graves, H.W. White, Z. Fisk, H.R. Ott, Solid State Commun. 36 861-868 (1980)

[9] N. Ali, S.B. Woods, J. Appl. Phys. 53 7905-7907 (1988)

[10] M. Takigawa, H. Yasuoka, T. Tanaka, Y. Ishizawa, J. Phys. Soc. Jpn. 52 728$731(1983)$

[11] I. Bat'ko, M. Bat'kova, K. Flachbart, D. Macko, E.S. Konovalova, Yu.B. Paderno, J. Magn. Magn. Mater. 140-144 1177-1178 (1995)

[12] Etoumeau, J.P. Mercurio, R. Naslain, P. Hagenmuller, J. Solid State Chem. 2 $332-343(1970)$

[13] R.W. Jonson, A.H. Daan, J. Chem. Phys 38 425-432 (1963)

[14] Y. Ishizava, T. Tanaka, E. Bannai, S. Kawai, J. Phys. Soc. Jpn. 42 112-117 (1997)

[15] F. Westrum, Les elements des terresrares, Paris, Centre Nat. Sci. 443-451 (1970)

[16] V.V. Novikov, Phys. Solid State 43(2) 300-304 (2001)

[17] T. Fugita, M. Suzuki, T. Komatsubara, S. Kunii, T. Kasuya, T. Ohtsuka, Solid State Commun. 35 569-572 (1980) 
[18] N.N Sirota, V.V. Novikov, V.A.Vinokurov, Yu.B. Paderno, Russ. J. Phys. Chem. A. 72 1785-1788 (1998)

[19] N.N. Sirota, V.V. Novikov, V.A. Vinokurov, L.A. Batova, Russ. J. Phys. Chem. A. 73 546-549 (1998)

[20] V.V. Novikov, Russ. J. Phys. Chem. A. 74 1550-1552 (2000)

[21] N.N. Sirota, V.V. Novikov, S.V. Antipov, Inorg. Mater. 34 907-909 (1998)

[22] N.N. Sirota, V.V. Novikov, S.V. Antipov, Phys. Solid State, 39 815-816 (1997)

[23] N.N. Sirota, V.V.Novikov, V.A.Vinokurov, Yu.B. Paderno, Phys. Solid State 40 1856-1858 (1998)

[24] N.N. Sirota, V.V. Novikov, Russ. J. Phys. Chem. A. 74 264-266 (2000)

[25] N.N.Sirota, V.V. Novikov, A.A. Sidorov, Phys. Solid State 42 199-200 (2000)

[26] N.N. Sirota, V.V. Novikov, J. Mater. Process. Manufacture. Sci. 7 111-114 (1988)

[27] N.N. Sirota, V.V. Novikov, A.V. Novikov, Phys. Solid State 42 2093-2096 (2000)

[28] H.R. Ott, B. Lüthi, Zeitschrift für Physik 28 141-147 (1977)

[29] H.R. Ott, B. Lüthi, Phys. Rev. Lett. 36 600-602 (1976)

[30] E. Zirngiebl, B. Hillebrands, S. Blumenroder, G. Guntherodt, M. Loewenhaupt et al., Phys, Rev. 30 4052-4054 (1984)

[31] M. Loewenhaupt, M. Prager, Zeitschrift für Physik 62 195-199 (1986) 